\title{
Treatment dropout in a family-based partial hospitalization program for eating disorders: a response to Kawada
}

\author{
Renee D. Rienecke ${ }^{1,2}$ (D) \\ Received: 18 February 2020 / Accepted: 23 February 2020 / Published online: 6 March 2020 \\ (c) Springer Nature Switzerland AG 2020
}

I appreciate Dr. Kawada's comment [1] regarding my article on treatment dropout in a family-based partial hospitalization program (PHP) [2], which found that lower percent of expected body weight at end of treatment was associated with treatment dropout of adolescents in the PHP. In addition, there was a trend towards patients who purged in the last month to have been less likely to have dropped out of treatment, and a trend towards patients who dropped out of treatment to have had fathers higher on the critical subscale of expressed emotion (EE).

The first study mentioned in Dr. Kawada's comment is a long-term study of outcomes in individuals with adolescentonset anorexia nervosa (AN) [3]. Dr. Kawada points out that treatment continuity and treatment dropout are associated with prognosis of $\mathrm{AN}$, but these factors were not assessed in the Dobrescu et al. study [3]. The second study reviewed by Dr. Kawada assessed parental EE and treatment outcome [4]. This study found that families with critical mothers were more likely to drop out of treatment, and patients who had critical fathers showed less improvement in eating disorder psychopathology. Maternal criticism was not associated with treatment dropout in the study commented on by Dr. Kawada [2], although paternal criticism was associated with dropout. It is unclear why maternal and paternal criticism would be associated with dropout in one study but not the other. However, the patient samples were quite different. In Rienecke [2], patients were in a partial hospitalization program for an average of 25 days, whereas in Rienecke et al. [4], patients participated in an outpatient randomized controlled trial over the course of 1 year. It is possible that different predictors

Renee D. Rienecke

renee.rienecke@ercinsight.com;

renee.rienecke@northwestern.edu

1 Eating Recovery Center, 333 N. Michigan Ave., Ste. 1900, Chicago, IL 60601, USA

2 Department of Psychiatry and Behavioral Sciences, Northwestern University, Chicago, IL 60611, USA of dropout are associated with different treatment settings and the differing degrees of parental involvement required in each, and Dr. Kawada points out that the authors conclude that maternal and paternal EE may have different impacts on treatment outcome [1, 4].

Dr. Kawada concludes with a recommendation to further explore factors associated with treatment dropout, particularly EE. EE has been shown to be a robust predictor of treatment dropout, treatment outcome, and relapse across a range of psychiatric and medical illnesses and certainly warrants further research attention.

Funding Not applicable.

\section{Compliance with ethical standards}

Conflict of interest Dr. Rienecke receives consulting fees from the Training Institute for Child and Adolescent Eating Disorders, LLC.

Ethical approval This article does not contain any studies with human participants or animals performed by any of the authors.

Informed consent For this type of study, formal consent is not required.

\section{References}

1. Kawada T (2020) Re: treatment dropout in a family-based partial hospitalization program for eating disorders. Eat Weight Disord. https://doi.org/10.1007/s40519-020-00870-2

2. Rienecke RD (2019) Treatment dropout in a family-based partial hospitalization program for eating disorders. Eat Weight Disord 24:163-168. https://doi.org/10.1007/s40519-018-0543-9

3. Dobrescu SR, Dinkler L, Gillberg C, Råstam M, Gillberg C, Wentz E (2020) Anorexia nervosa: 30-year outcome. Br J Psychiatry 216:97-104. https://doi.org/10.1192/bjp.2019.113

4. Rienecke RD, Accurso EC, Lock J, Le Grange D (2016) Expressed emotion, family functioning, and treatment outcome for adolescents with anorexia nervosa. Eur Eat Disord Rev 24:43-51. https ://doi.org/10.1002/erv.2389

Publisher's Note Springer Nature remains neutral with regard to jurisdictional claims in published maps and institutional affiliations. 